\title{
Low-regret control for a fractional wave equation with incomplete data
}

\author{
Dumitru Baleanu ${ }^{1,2}$, Claire Joseph ${ }^{3}$ and Gisèle Mophou $3,4^{*}$ (i)
}

${ }^{*}$ Correspondence:

gmophou@univ-ag.fr

${ }^{3}$ Université des Antilles et de la

Guyane, Campus Fouillole, 97159

Pointe-à-Pitre, Guadeloupe (FWI),

France

${ }^{4}$ Laboratoire MAINEGE, Université

Ouaga 3S, 06 BP 10347

Ouagadougou 06, Burkina Faso

Full list of author information is

available at the end of the article

\begin{abstract}
We investigate in this manuscript an optimal control problem for a fractional wave equation involving the fractional Riemann-Liouville derivative and with missing initial condition. For this purpose, we use the concept of no-regret and low-regret controls. Assuming that the missing datum belongs to a certain space we show the existence and the uniqueness of the low-regret control. Besides, its convergence to the no-regret control is discussed together with the optimality system describing the no-regret control.
\end{abstract}

Keywords: Riemann-Liouville fractional derivative; Caputo fractional derivative; optimal control; no-regret control; low-regret control

\section{Introduction}

Let us consider $N \in \mathbb{N}^{*}$ and $\Omega$ a bounded open subset of $\mathbb{R}^{N}$ possessing the boundary $\partial \Omega$ of class $C^{2}$. When the time $T>0$, we consider $\left.Q=\Omega \times\right] 0, T[$ and $\Sigma=\partial \Omega \times] 0, T[$ and we discuss the fractional wave equation:

$$
\left\{\begin{array}{l}
D_{\mathrm{RL}}^{\alpha} y(x, t)-\Delta y(x, t)=v(x, t), \quad(x, t) \in Q \\
y(\sigma, t)=0, \quad(\sigma, t) \in \Sigma \\
I^{2-\alpha} y\left(x, 0^{+}\right)=y^{0}, \quad x \in \Omega \\
\frac{\partial}{\partial t} I^{2-\alpha} y\left(x, 0^{+}\right)=g, \quad x \in \Omega
\end{array}\right.
$$

such that $3 / 2<\alpha<2, y^{0} \in H^{2}(\Omega) \cap H_{0}^{1}(\Omega), I^{2-\alpha} y\left(x, 0^{+}\right)=\lim _{t \rightarrow 0} I^{2-\alpha} y(x, t)$ and $\frac{\partial}{\partial t} I^{2-\alpha} y(x$, $\left.0^{+}\right)=\lim _{t \rightarrow 0} \frac{\partial}{\partial t} I^{2-\alpha} y(x, t)$ where the fractional integral $I^{\alpha}$ of order $\alpha$ and the fractional derivative $D_{\mathrm{RL}}^{\alpha}$ of order $\alpha$ are within the Riemann-Liouville sense. The function $g$ is unknown and belongs to $L^{2}(\Omega)$ and the control $v \in L^{2}(Q)$.

Since the initial condition is unknown, the system (1) is a fractional wave equations with missing data. Such equations are used to model pollution phenomena. In this system $g$ represents the pollution term.

According to the data, we know that system (1) admits a unique solution $y(v, g)=$ $y(x, t ; v, g)$ in $L^{2}\left((0, T) ; H_{0}^{1}(\Omega)\right) \subset L^{2}(Q)[1]$. Hence, we can define the following functional:

$$
J(v, g)=\left\|y(v, g)-z_{d}\right\|_{L^{2}(Q)}^{2}+N\|v\|_{L^{2}(Q)}^{2},
$$

where $z_{d} \in L^{2}(Q)$ and $N>0$.

(c) 2016 Baleanu et al. This article is distributed under the terms of the Creative Commons Attribution 4.0 International License (http://creativecommons.org/licenses/by/4.0/), which permits unrestricted use, distribution, and reproduction in any medium, provided you give appropriate credit to the original author(s) and the source, provide a link to the Creative Commons license, and indicate if changes were made. 
In this manuscript, we discuss the optimal control problem, namely

$$
\inf _{v \in L^{2}(Q)} J(v, g), \quad \forall g \in L^{2}(\Omega)
$$

If the function $g$ is given, namely $g=g_{0} \in L^{2}(\Omega)$, then system (1) is completely determined and problem (3) becomes a classical optimal control problem [2]. Such a problem was studied by Mophou and Joseph [1] with a cost function defined with a final observation. Actually, the authors proved that one can approach the fractional integral of order $0<$ $2-\alpha<1 / 2$ of the state at final time by a desired state by acting on a distributed control. For more literature on fractional optimal control, we refer to [3-11] and the references therein.

Since the function $g$ is unknown, the optimal control problem (3) has no sense because $L^{2}(\Omega)$ is of infinite dimension. So, to solve this problem, we proceed as Lions [12,13] for the control of partial differential equations with integer time derivatives and missing data. This means that we use the notions of no-regret and low-regret controls. There are many works using these concepts in the literature. In [14] for instance, Nakoulima et al. utilized these concepts to control distributed linear systems possessing missing data. A generalization of this approach can be found in [15] for some nonlinear distributed systems possessing incomplete data. Jacob and Omrane used the notion of no-regret control to control a linear population dynamics equation with missing initial data [16]. Recently, Mophou [17] used these notions to control a fractional diffusion equation with unknown boundary condition. For more literature on such control we refer to [18-23] and the references therein.

In our paper, we show that the low-regret control problem associated to (1) admits a unique solution which converges toward the no-regret control. We provide the singular optimality system for the no-regret control.

Below we present the organization of our manuscript. In the following section, we show briefly some results about fractional derivatives and preliminary results on the existence and uniqueness of solution to fractional wave equations. In Section 3, we investigate the no-regret and low-regret control problems corresponding to (1).

\section{Preliminaries}

Below, we give briefly some results about fractional calculus and some existence results about fractional wave equations.

Definition 2.1 [24, 25] If $f: \mathbb{R}_{+} \rightarrow \mathbb{R}$ is a continuous function on $\mathbb{R}_{+}$, and $\alpha>0$, then the expression of the Riemann-Liouville fractional integral of order $\alpha$ is

$$
I^{\alpha} f(t)=\frac{1}{\Gamma(\alpha)} \int_{0}^{t}(t-s)^{\alpha-1} f(s) d s, \quad t>0 .
$$

Definition 2.2 $[25,26]$ The form of the left Riemann-Liouville fractional derivative of order $0 \leq n-1<\alpha<n, n \in \mathbb{N}$ of $f$ is given by

$$
D_{\mathrm{RI}}^{\alpha} f(t)=\frac{1}{\Gamma(n-\alpha)} \cdot \frac{d^{n}}{d t^{n}} \int_{0}^{t}(t-s)^{n-1-\alpha} f(s) d s, \quad t>0
$$


Definition $2.3[25,26]$ The left Caputo fractional derivative of order $0 \leq n-1<\alpha<n$, $n \in \mathbb{N}$ of $f$ is given by

$$
D_{\mathrm{C}}^{\alpha} f(t)=\frac{1}{\Gamma(n-\alpha)} \int_{0}^{t}(t-s)^{n-1-\alpha} f^{(n)}(s) d s, \quad t>0 .
$$

We mention that in the above two definitions we consider $f: \mathbb{R}_{+} \rightarrow \mathbb{R}$.

Definition 2.4 [25-27] Let $f: \mathbb{R}_{+} \rightarrow \mathbb{R}, 0 \leq n-1<\alpha<n, n \in \mathbb{N}$. Then the right Caputo fractional derivative of order $\alpha$ of $f$ is

$$
\mathcal{D}_{\mathrm{C}}^{\alpha} f(t)=\frac{(-1)^{n}}{\Gamma(n-\alpha)} \int_{t}^{T}(s-t)^{n-1-\alpha} f^{(n)}(s) d s, \quad 0<t<T .
$$

In all above definitions we assume that the integrals exist.

Lemma $2.5[1]$ Let $y \in \mathcal{C}^{\infty}(\bar{Q})$ and $\varphi \in \mathcal{C}^{\infty}(\bar{Q})$. Then we have

$$
\begin{aligned}
\int_{Q}\left(D_{\mathrm{RL}}^{\alpha} y(x, t)-\Delta y(x, t)\right) \varphi(x, t) d x d t \\
=\int_{\Omega} \varphi(x, T) \frac{\partial}{\partial t} I^{2-\alpha} y(x, T) d x-\int_{\Omega} \varphi(x, 0) \frac{\partial}{\partial t} I^{2-\alpha} y\left(x, 0^{+}\right) d x \\
\quad-\int_{\Omega} I^{2-\alpha} y(x, T) \frac{\partial \varphi}{\partial t}(x, T) d x+\int_{\Omega} I^{2-\alpha} y(x, 0) \frac{\partial \varphi}{\partial t}(x, 0) d x \\
\quad+\int_{\Sigma} y(\sigma, s) \frac{\partial \varphi}{\partial v}(\sigma, s) d \sigma d t-\int_{\Sigma} \frac{\partial y}{\partial v}(\sigma, s) \varphi(\sigma, s) d \sigma d t \\
\quad+\int_{Q} y(x, t)\left(\mathcal{D}_{\mathrm{C}}^{\alpha} \varphi(x, t)-\Delta \varphi(x, t)\right) d x d t .
\end{aligned}
$$

In the following we give some results that will be use to prove the existence of the lowregret and no-regret controls.

Theorem 2.6 [1] Let $3 / 2<\alpha<2, y^{0} \in H^{2}(\Omega) \cap H_{0}^{1}(\Omega), y^{1} \in L^{2}(\Omega)$ and $v \in L^{2}(Q)$. Then the problem

$$
\left\{\begin{array}{l}
D_{\mathrm{RL}}^{\alpha} y(x, t)-\Delta y(x, t)=v(x, t), \quad(x, t) \in Q \\
y(\sigma, t)=0, \quad(\sigma, t) \in \Sigma \\
I^{2-\alpha} y\left(x, 0^{+}\right)=y^{0}, \quad x \in \Omega \\
\frac{\partial}{\partial t} I^{2-\alpha} y\left(x, 0^{+}\right)=y^{1}, \quad x \in \Omega
\end{array}\right.
$$

has a unique solution $y \in L^{2}\left((0, T) ; H_{0}^{1}(\Omega)\right)$. Moreover, the following estimates hold:

$$
\begin{aligned}
& \|y\|_{L^{2}\left((0, T) ; H_{0}^{1}(\Omega)\right)} \leq \Delta\left(\left\|y^{0}\right\|_{H_{0}^{1}(\Omega)}+\left\|y^{1}\right\|_{L^{2}(\Omega)}+\|v\|_{L^{2}(Q)}\right), \\
& \left\|I^{2-\alpha} y\right\|_{C\left([0, T] ; H_{0}^{1}(\Omega)\right)} \leq \Pi\left(\left\|y^{0}\right\|_{H_{0}^{1}(\Omega)}+\left\|y^{1}\right\|_{L^{2}(\Omega)}+\|v\|_{L^{2}(Q)}\right), \\
& \left\|\frac{\partial}{\partial t} I^{2-\alpha} y\right\|_{C\left([0, T] ; L^{2}(\Omega)\right)} \leq \Theta\left(\left\|y^{0}\right\|_{H^{2}(\Omega)}+\left\|y^{1}\right\|_{L^{2}(\Omega)}+\|v\|_{L^{2}(Q)}\right),
\end{aligned}
$$


with

$$
\begin{aligned}
& \Delta=\max \left(C \sqrt{\frac{2 T^{2 \alpha-3}}{(2 \alpha-3)}}, C \sqrt{\frac{T^{\alpha-1}}{(\alpha-1)}}, C \sqrt{\frac{2 T^{\alpha}}{\alpha(\alpha-1)}}\right), \\
& \Pi=\sup \left(C \sqrt{2}, C \sqrt{2 T^{2-\alpha}}, C \sqrt{\frac{2 T^{3-\alpha}}{(3-\alpha)}}\right),
\end{aligned}
$$

and

$$
\Theta=\max \left(\sqrt{2} C T^{\alpha-1}, \sqrt{2} C\right)
$$

Consider the fractional wave equation involving the left Caputo fractional derivative of order $3 / 2<\alpha<2$ :

$$
\left\{\begin{array}{l}
D_{\mathrm{C}}^{\alpha} y(x, t)-\Delta y(x, t)=f, \quad(x, t) \in Q \\
y(\sigma, t)=0, \quad(\sigma, t) \in \Sigma \\
y(x, 0)=0, \quad x \in \Omega \\
\frac{\partial y}{\partial t}(x, 0)=0, \quad x \in \Omega
\end{array}\right.
$$

where $f \in L^{2}(Q)$.

Theorem 2.7 Let $f \in L^{2}(Q)$. Then problem (11) has a unique solution $y \in C\left([0, T] ; H_{0}^{1}(\Omega)\right)$. Moreover, $\frac{\partial y}{\partial t} \in C\left([0, T] ; L^{2}(\Omega)\right)$ and there exists $C>0$ in such a way that

$$
\|y\|_{C\left([0, T] ; H_{0}^{1}(\Omega)\right)} \leq C \sqrt{\frac{T^{\alpha-1}}{\alpha-1}}\|f\|_{L^{2}(Q)}
$$

and

$$
\left\|\frac{\partial y}{\partial t}\right\|_{C\left([0, T] ; L^{2}(\Omega)\right)} \leq C \sqrt{\frac{T^{2 \alpha-3}}{2 \alpha-3}}\|f\|_{L^{2}(Q)} .
$$

Proof Below we proceed as was mentioned in [28].

Corollary 2.8 Let $3 / 2<\alpha<2$ and $\phi \in L^{2}(Q)$. Consider the fractional wave equation:

$$
\left\{\begin{array}{l}
\mathcal{D}_{\mathrm{C}}^{\alpha} \psi(x, t)-\Delta \psi(x, t)=\phi, \quad(x, t) \in \mathcal{Q}, \\
\psi(\sigma, t)=0, \quad(\sigma, t) \in \Sigma, \\
\psi(x, T)=0, \quad x \in \Omega \\
\frac{\partial \psi}{\partial t}(x, T)=0, \quad x \in \Omega,
\end{array}\right.
$$

where $\mathcal{D}_{C}^{\alpha}$ is the right Caputo fractional derivative of order $1<\alpha<2$. Then (14) has a unique solution $\psi \in \mathcal{C}\left([0, T] ; H_{0}^{1}(\Omega)\right)$. Moreover, $\frac{\partial \psi}{\partial t} \in C\left([0, T] ; L^{2}(\Omega)\right)$, and there exists $C>0$ fulfilling

$$
\|\psi\|_{C\left([0, T] ; H_{0}^{1}(\Omega)\right)} \leq C \sqrt{\frac{T^{\alpha-1}}{\alpha-1}}\|\phi\|_{L^{2}(Q)}
$$


and

$$
\left\|\frac{\partial \psi}{\partial t}\right\|_{C\left([0, T] ; L^{2}(\Omega)\right)} \leq C \sqrt{\frac{T^{2 \alpha-3}}{2 \alpha-3}}\|\phi\|_{L^{2}(Q)} .
$$

Proof If we make the change of variable $t \rightarrow T-t$ in (14), then we conclude that $\hat{\psi}(t)=$ $\psi(T-t)$ verifies

$$
\left\{\begin{array}{l}
D_{\mathrm{C}}^{\alpha} \hat{\psi}-\Delta \hat{\psi}=\hat{\phi} \quad \text { in } Q \\
\hat{\psi}=0 \text { on } \Sigma \\
\hat{\psi}(0)=0 \quad \text { in } \Omega \\
\frac{\partial \hat{\psi}}{\partial t}(0)=0 \text { in } \Omega
\end{array}\right.
$$

where $\hat{\phi}(t)=\phi(T-t)$ and $D_{\mathrm{C}}^{\alpha}$ is the left Caputo fractional derivative of order $3 / 2<\alpha<2$. Because $T-t \in[0, T]$ when $t \in[0, T]$, we say that $\hat{\phi} \in L^{2}(Q)$ due to the fact that $\phi \in L^{2}(Q)$. It then suffices to use Theorem 2.7 to conclude.

We also need some trace results.

Lemma 2.9 [1] Let $f \in L^{2}(Q)$ and $y \in L^{2}(Q)$ such that $D_{\mathrm{RL}}^{\alpha} y-\Delta y=f$. Then:

(i) $y_{\mid \partial \Omega}$ and $\frac{\partial y}{\partial v}{ }_{\mid \partial \Omega}$ exist and belong to $H^{-2}\left((0, T) ; H^{-1 / 2}(\partial \Omega)\right)$ and $H^{-2}\left((0, T) ; H^{-3 / 2}(\partial \Omega)\right)$ respectively.

(ii) $I^{2-\alpha} y \in C\left([0, T] ; L^{2}(\Omega)\right)$.

(iii) $\frac{\partial}{\partial t} I^{2-\alpha} y \in C\left([0, T] ; H^{-1}(\Omega)\right)$.

\section{Existence and uniqueness of no-regret and low-regret controls}

Below, we show the existence and the uniqueness of the no-regret control and the lowregret control problem for system (1).

Lemma 3.1 Let $v \in L^{2}(Q)$ and $g \in L^{2}(\Omega)$. Then we have

$$
\begin{aligned}
J(v, g)= & J(0, g)+J(v, 0)-J(0,0) \\
& +2 \int_{Q}[y(0, g)-y(0,0)][y(v, 0)-y(0,0)] d t d x .
\end{aligned}
$$

Here J denotes the functional given by (2) and $y(v, g)=y(x, t ; v, g) \in L^{2}\left(0, T ; H_{0}^{1}(\Omega)\right) \subset L^{2}(Q)$ is the solution of (1).

Proof Let us consider $y(v, 0)=y(x, t ; v, 0), y(0, g)=y(x, t ; 0, g)$, and $y(0,0)=y(x, t ; 0,0)$ be the solutions of

$$
\begin{aligned}
& \left\{\begin{array}{l}
D_{\mathrm{RL}}^{\alpha} y(v, 0)-\Delta y(v, 0)=v \quad \text { in } Q \\
y=0 \quad \text { on } \Sigma \\
I^{2-\alpha} y(0 ; v, 0)=y^{0} \quad \text { in } \Omega \\
\frac{\partial}{\partial t} I^{2-\alpha} y(0 ; v, 0)=0 \quad \text { in } \Omega
\end{array}\right. \\
& \begin{cases}D_{\mathrm{RL}}^{\alpha} y(0, g)-\Delta y(0, g)=0 \\
y=0 \quad \text { on } \Sigma, \\
I^{2-\alpha} y(0 ; 0, g)=y^{0} \quad \text { in } \Omega \\
\frac{\partial}{\partial t} I^{2-\alpha} y(0 ; 0, g)=g & \text { in } \Omega\end{cases}
\end{aligned}
$$


and

$$
\left\{\begin{array}{l}
D_{\mathrm{RL}}^{\alpha} y(0,0)-\Delta y(0,0)=0 \quad \text { in } Q, \\
y=0 \text { on } \Sigma, \\
I^{2-\alpha} y(0 ; 0,0)=y^{0} \quad \text { in } \Omega, \\
\frac{\partial}{\partial t} I^{2-\alpha} y(0 ; 0,0)=0 \quad \text { in } \Omega,
\end{array}\right.
$$

where $I^{2-\alpha} y(0 ; v, g)=\lim _{t \rightarrow 0^{+}} I^{2-\alpha} y(x, t ; v, g)$ and $\frac{\partial}{\partial t} I^{2-\alpha} y(0 ; v, g)=\lim _{t \rightarrow 0^{+}} \frac{\partial}{\partial t} I^{2-\alpha} y(x, t ; v, g)$.

Since $v \in L^{2}(Q), y^{0} \in H_{0}^{1}(\Omega) \cap H^{2}(\Omega)$ and $g \in L^{2}(\Omega)$, we see from Theorem 2.6 that $y(v, 0)$, $y(0, g)$ and $y(0,0)$ belong to $L^{2}\left((0, T) ; H_{0}^{1}(\Omega)\right)$.

Observing that

$$
\begin{aligned}
& J(v, 0)=\int_{Q}\left[y(v, 0)-z_{d}\right]\left[y(v, 0)-z_{d}\right] d t d x+N\|v\|_{L^{2}(Q)}^{2}, \\
& J(0, g)=\int_{Q}\left[y(0, g)-z_{d}\right]\left[y(0, g)-z_{d}\right] d t d x, \\
& J(0,0)=\int_{Q}\left[y(0,0)-z_{d}\right]\left[y(0,0)-z_{d}\right] d t d x,
\end{aligned}
$$

and using the fact that

$$
y(v, g)=y(v, 0)+y(0, g)-y(0,0),
$$

we have

$$
\begin{aligned}
J(v, g)= & \left\|y(v, g)-z_{d}\right\|_{L^{2}(Q)}^{2}+N\|v\|_{L^{2}(Q)}^{2} \\
= & \left\|y(v, 0)+y(0, g)-y(0,0)-z_{d}\right\|_{L^{2}(Q)}^{2}+N\|v\|_{L^{2}(Q)}^{2} \\
= & J(v, 0)+2 \int_{Q}\left[y(v, 0)-z_{d}\right][y(0, g)-y(0,0)] d t d x \\
& +\left\|y(0, g)-y(0,0)-z_{d}\right\|_{L^{2}(Q)}^{2} \\
= & J(v, 0)+2 \int_{Q}[y(v, 0)-y(0,0)][y(0, g)-y(0,0)] d t d x \\
& +2 \int_{Q}\left[y(0,0)-z_{d}\right][y(0, g)-y(0,0)] d t d x+\left\|y(0, g)-y(0,0)-z_{d}\right\|_{L^{2}(Q)}^{2} .
\end{aligned}
$$

Using

$$
\begin{aligned}
\left\|y(0, g)-y(0,0)-z_{d}\right\|_{L^{2}(Q)}^{2}= & J(0, g)+J(0,0) \\
& -2 \int_{Q}\left[y(0,0)-z_{d}\right][y(0, g)-y(0,0)] d t d x-2 J(0,0),
\end{aligned}
$$

we conclude that

$$
\begin{aligned}
J(v, g)= & J(0, g)+J(v, 0)-J(0,0) \\
& +2 \int_{\Omega} \int_{0}^{T}[y(v, 0)-y(0,0)][y(0, g)-y(0,0)] d t d x .
\end{aligned}
$$


Lemma 3.2 Let $v \in L^{2}(Q)$ and $g \in L^{2}(\Omega)$. Then we have

$$
J(v, g)=J(0, g)+J(v, 0)-J(0,0)+2 \int_{\Omega} g \zeta(x, 0 ; v) d x,
$$

where $\zeta(v)=\zeta(x, t ; v) \in C\left([0, T] ; H_{0}^{1}(\Omega)\right)$ be solution of

$$
\left\{\begin{array}{l}
\mathcal{D}_{\mathrm{C}}^{\alpha} \zeta(v)-\Delta \zeta(v)=y(v, 0)-y(0,0) \quad \text { in } Q \\
\zeta=0 \text { on } \Sigma, \\
\zeta(x, T ; v)=0 \quad \text { in } \Omega \\
\frac{\partial \zeta}{\partial t}(x, T ; v)=0 \quad \text { in } \Omega
\end{array}\right.
$$

Proof Since $y(v, 0)-y(0,0) \in L^{2}(Q)$, from Proposition 2.8, we know that the system (24) admits a unique solution $\zeta(v) \in C\left([0, T] ; H_{0}^{1}(\Omega)\right)$. Also, there exists $C>0$ such that

$$
\|\zeta(v)\|_{C\left([0, T] ; H_{0}^{1}(\Omega)\right)} \leq C \sqrt{\frac{T^{\alpha-1}}{\alpha-1}}\|y(v, 0)-y(0,0)\|_{L^{2}(Q)}
$$

and

$$
\left\|\frac{\partial \zeta}{\partial t}(v)\right\|_{\mathcal{C}\left([0, T] ; L^{2}(\Omega)\right)} \leq C \sqrt{\frac{T^{2 \alpha-3}}{2 \alpha-3}}\|y(v, 0)-y(0,0)\|_{L^{2}(Q)} .
$$

Set $z=y(g, 0)-y(0,0)$. Then $z$ verifies

$$
\left\{\begin{array}{l}
D_{\mathrm{RL}}^{\alpha} z-\Delta z=0 \text { in } Q \\
z=0 \text { on } \Sigma \\
I^{2-\alpha} z(0)=0 \text { in } \Omega \\
\frac{\partial}{\partial t} I^{2-\alpha} z(0)=g \text { in } \Omega
\end{array}\right.
$$

Since $g \in L^{2}(\Omega)$, it follows from Theorem 2.6 that $z \in L^{2}\left((0, T) ; H_{0}^{1}(\Omega)\right), I^{2-\alpha} z \in C([0, T]$, $\left.H_{0}^{1}(\Omega)\right)$, and $\frac{\partial}{\partial t} I^{2-\alpha} z \in C\left([0, T], L^{2}(\Omega)\right)$. So, if we multiply the first equation of (24) by $z$ utilizing the fractional integration by parts provided by Lemma 2.5 , we conclude

$$
\begin{aligned}
& \int_{Q}(y(v, 0)-y(0,0)) z d t d x \\
& =\int_{Q}\left(\mathcal{D}_{C}^{\alpha} \zeta(v)-\Delta \zeta(v)\right) z d t d x \\
& =\int_{\Omega} \zeta(x, 0 ; v) \frac{\partial}{\partial t} I^{2-\alpha} z(0) d x .
\end{aligned}
$$

Thus, replacing $z$ by $(y(0, g)-y(0,0))$, we obtain

$$
\int_{Q}[y(v, 0)-y(0,0)][y(0, g)-y(0,0)] d t d x=\int_{\Omega} \zeta(x, 0 ; v) g d x,
$$

and (18) becomes

$$
J(v, g)-J(0, g)=J(v, 0)-J(0,0)+2 \int_{\Omega} \zeta(x, 0 ; v) g d x .
$$


Now we consider the no-regret control problem:

$$
\inf _{v \in L^{2}(Q)} \sup _{g \in L^{2}(\Omega)}(J(v, g)-J(0, g))
$$

From (23), this problem is equivalent to the following one:

$$
\inf _{v \in L^{2}(Q)} \sup _{g \in L^{2}(\Omega)}\left[J(v, 0)-J(0,0)+2 \int_{\Omega} \zeta(x, 0 ; v) g d x\right] .
$$

As the space $L^{2}(\Omega)$ is a vector space, the no-regret control exists only if

$$
\sup _{g \in L^{2}(\Omega)}\left(\int_{\Omega} \zeta(x, 0 ; v) g d x\right)=0
$$

This implies that the no-regret control belongs to $\mathcal{U}$ defined by

$$
\mathcal{U}=\left\{v \in L^{2}(Q) \mid\left(\int_{\Omega} \zeta(x, 0 ; v) g d x\right)=0, \forall g \in L^{2}(\Omega)\right\} .
$$

As a result such control should be carefully investigated. So, we proceed by penalization. For all $\gamma>0$, we discuss the low-regret control problem:

$$
\inf _{v \in L^{2}(Q)} \sup _{g \in L^{2}(\Omega)}\left(J(v, g)-J(0, g)-\gamma\|g\|_{L^{2}(\Omega)}^{2}\right) .
$$

According to (23), the problem (32) is equivalent to the following problem:

$$
\inf _{v \in L^{2}(Q)}\left[J(v, 0)-J(0,0)+2 \sup _{g \in L^{2}(\Omega)}\left(\int_{\Omega} \zeta(x, 0 ; v) g d x-\frac{\gamma}{2}\|g\|_{L^{2}(\Omega)}^{2}\right)\right] .
$$

Using the Legendre-Fenchel transform, we conclude

$$
2 \gamma \sup _{g \in L^{2}(\Omega)}\left(\int_{\Omega} \frac{1}{\gamma} \zeta(x, 0 ; v) g d x-\frac{1}{\gamma} \frac{\gamma}{2}\|g\|_{L^{2}(\Omega)}^{2}\right)=\frac{1}{\gamma}\|\zeta(\cdot, 0 ; v)\|_{L^{2}(\Omega)}^{2},
$$

and problem (32) becomes: For any $\gamma>0$, find $u^{\gamma} \in L^{2}(Q)$ such that

$$
J_{\gamma}\left(u^{\gamma}\right)=\inf _{v \in L^{2}(Q)} J_{\gamma}(v)
$$

where

$$
J_{\gamma}(v)=J(v, 0)-J(0,0)+\frac{1}{\gamma}\|\zeta(\cdot, 0 ; v)\|_{L^{2}(\Omega)}^{2} .
$$

Proposition 3.3 Let $\gamma>0$. Then (33) has a unique solution $u^{\gamma}$, called a low-regret control.

Proof We recall that

$$
J_{\gamma}(v)=J(v, 0)-J(0,0)+\frac{1}{\gamma}\|\zeta(\cdot, 0 ; v)\|_{L^{2}(\Omega)}^{2} \geq-J(0,0) .
$$


Thus, we can say that $\inf _{v \in L^{2}(Q)} J_{\gamma}$ exists. Let $\left(v_{n}\right) \in L^{2}(Q)$ be a minimizing sequence such that

$$
\lim _{n \rightarrow+\infty} J_{\gamma}\left(v_{n}\right)=\inf _{v \in L^{2}(Q)} J_{\gamma}(v)
$$

Then $y_{n}=y\left(x, t ; v_{n}, 0\right)$ is a solution of $(19)$ and $y_{n}$ satisfies

$$
\begin{aligned}
& D_{\mathrm{RL}}^{\alpha} y_{n}(x, t)-\Delta y_{n}(x, t)=v_{n}(x, t) \text { in } Q, \\
& y_{n}(x, t)=0 \quad \text { on } \Sigma, \\
& I^{2-\alpha} y_{n}(x, 0)=y^{0} \quad \text { in } \Omega, \\
& \frac{\partial}{\partial t} I^{2-\alpha} y_{n}(x, 0)=0 \quad \text { in } \Omega .
\end{aligned}
$$

It follows from (35) that there exists $C(\gamma)>0$ independent of $n$ such that

$$
0 \leq J\left(v_{n}, 0\right)+\frac{1}{\gamma}\left\|\zeta\left(\cdot, 0 ; v_{n}\right)\right\|_{L^{2}(\Omega)}^{2} \leq C(\gamma)+J(0,0)=C(\gamma) .
$$

From the definition of $J\left(v_{n}, 0\right)$ we obtain

$$
\begin{aligned}
& \left\|v_{n}\right\|_{L^{2}(Q)} \leq C(\gamma), \\
& \left\|\zeta\left(\cdot, 0 ; v_{n}\right)\right\|_{L^{2}(\Omega)} \leq \sqrt{\gamma} C(\gamma) .
\end{aligned}
$$

Therefore, from Theorem 2.6, we know that there exists a constant $C$ independent of $n$ such that

$$
\begin{aligned}
& \left\|y_{n}\right\|_{L^{2}\left((0, T) ; H_{0}^{1}(\Omega)\right)} \leq C(\gamma), \\
& \left\|I^{2-\alpha} y_{n}\right\|_{L^{2}\left((0, T) ; H_{0}^{1}(\Omega)\right)} \leq C(\gamma), \\
& \left\|\frac{\partial}{\partial t} I^{2-\alpha} y_{n}\right\|_{L^{2}\left((0, T) ; L^{2}(\Omega)\right)} \leq C(\gamma) .
\end{aligned}
$$

Moreover, from (36a) and (37a), we have

$$
\left\|D_{\mathrm{RL}}^{\alpha} y_{n}-\Delta y_{n}\right\|_{L^{2}(Q)} \leq C(\gamma) \text {. }
$$

Consequently, there exist $u^{\gamma} \in L^{2}(Q), y^{\gamma} \in L^{2}\left((0, T) ; H_{0}^{1}(\Omega)\right), \delta \in L^{2}(Q), \eta \in L^{2}((0, T)$; $\left.H_{0}^{1}(\Omega)\right), \theta \in L^{2}\left((0, T) ; L^{2}(\Omega)\right)$ and we can extract subsequences of $\left(v_{n}\right)$ and $\left(y_{n}\right)$ (still called $\left(v_{n}\right)$ and $\left.\left(y_{n}\right)\right)$ such that:

$$
\begin{aligned}
& v_{n} \rightarrow u^{\gamma} \quad \text { weakly in } L^{2}(Q), \\
& D_{\mathrm{RL}}^{\alpha} y_{n}-\Delta y_{n} \rightarrow \delta \quad \text { weakly in } L^{2}(Q), \\
& y_{n} \rightarrow y^{\gamma} \quad \text { weakly in } L^{2}\left((0, T) ; H_{0}^{1}(\Omega)\right), \\
& I^{2-\alpha} y_{n} \rightarrow \eta \quad \text { weakly in } L^{2}\left([0, T], H_{0}^{1}(\Omega)\right), \\
& \frac{\partial}{\partial t} I^{2-\alpha} y_{n} \rightarrow \theta \quad \text { weakly in } L^{2}\left((0, T) ; L^{2}(\Omega)\right) .
\end{aligned}
$$


The remaining part of the proof contains three steps.

Step 1: We show that $\left(u^{\gamma}, y^{\gamma}\right)$ fulfills (19).

Set $\mathbb{D}(Q)$, the set of $\mathcal{C}^{\infty}$ function on $Q$ with compact support and denote by $\mathbb{D}^{\prime}(Q)$ its dual. Multiplying (36a) by $\varphi \in \mathbb{D}(Q)$ and using Lemma 2.5, (40a), and (40c), we prove as in [1] that

$$
D_{\mathrm{RL}}^{\alpha} y_{n}-\Delta y_{n} \rightarrow D_{\mathrm{RL}}^{\alpha} y^{\gamma}-\Delta y^{\gamma} \quad \text { weakly in } \mathbb{D}^{\prime}(Q) \text {. }
$$

From (40b) and the uniqueness of the limit, we conclude

$$
D_{\mathrm{RL}}^{\alpha} y^{\gamma}-\Delta y^{\gamma}=\delta \in L^{2}(Q)
$$

Hence,

$$
D_{\mathrm{RL}}^{\alpha} y_{n}-\Delta y_{n} \rightarrow D_{\mathrm{RL}}^{\alpha} y^{\gamma}-\Delta y^{\gamma} \quad \text { weakly in } L^{2}(Q) .
$$

Then passing to the limit in (36a) and using (41) and (40a), we obtain

$$
D_{\mathrm{RL}}^{\alpha} y^{\gamma}(x, t)-\Delta y^{\gamma}(x, t)=u^{\gamma}(x, t), \quad(x, t) \in Q
$$

On the other hand, we have

$$
\begin{aligned}
& \int_{Q} I^{2-\alpha} y_{n}(x, t) \varphi(x, t) d t d x \\
& \quad=\int_{\Omega} \int_{0}^{T} y_{n}(x, s)\left(\frac{1}{\Gamma(2-\alpha)} \int_{s}^{T}(t-s)^{1-\alpha} \varphi(x, t) d t\right) d s d x, \quad \forall \varphi \in \mathbb{D}(Q) .
\end{aligned}
$$

Thus using (40c) and (40d), while passing to the limit, we get

$$
\begin{aligned}
\int_{Q} \eta \varphi(x, t) d t d x & =\int_{\Omega} \int_{0}^{T} y^{\gamma}(x, s)\left(\frac{1}{\Gamma(2-\alpha)} \int_{s}^{T}(t-s)^{1-\alpha} \varphi(x, t) d t\right) d s d x \\
& =\int_{Q} I^{2-\alpha} y^{\gamma}(x, t) \varphi(x, t) d t d x, \quad \forall \varphi \in \mathbb{D}(Q) .
\end{aligned}
$$

This implies that

$$
I^{2-\alpha} y^{\gamma}(x, t)=\eta \quad \text { in } Q .
$$

Thus, (40d) becomes

$$
I^{2-\alpha} y_{n} \rightarrow I^{2-\alpha} y^{\gamma} \quad \text { weakly in } L^{2}\left([0, T], H_{0}^{1}(\Omega)\right) .
$$

In view of (44), we have

$$
\frac{\partial}{\partial t} I^{2-\alpha} y_{n} \rightarrow \frac{\partial}{\partial t} I^{2-\alpha} y^{\gamma} \quad \text { weakly in } \mathbb{D}^{\prime}(Q)
$$


and as we have (40e), we obtain

$$
\frac{\partial}{\partial t} I^{2-\alpha} y_{n} \rightarrow \frac{\partial}{\partial t} I^{2-\alpha} y^{\gamma}=\theta \quad \text { weakly in } L^{2}(Q)
$$

Since $y^{\gamma} \in L^{2}(Q)$ and $D_{\mathrm{RL}}^{\alpha} y^{\gamma}-\Delta y^{\gamma} \in L^{2}(Q)$, in view of Lemma 2.9, we know that $y_{\mid \partial \Omega}^{\gamma}$ and $\frac{\partial y^{\gamma}}{\partial \nu} \mid \partial \Omega$ exist and belong to $H^{-2}\left((0, T) ; H^{-1 / 2}(\partial \Omega)\right)$ and $H^{-2}\left((0, T) ; H^{-3 / 2}(\partial \Omega)\right)$, respectively. Moreover, we have $I^{2-\alpha} y^{\gamma} \in C\left([0, T] ; L^{2}(\Omega)\right)$ and $\frac{\partial}{\partial t} I^{2-\alpha} y^{\gamma} \in C\left([0, T] ; H^{-1}(\Omega)\right)$.

Now multiplying (36a) by a function $\varphi \in C^{\infty}(\bar{Q})$ such that $\varphi_{\mid \partial \Omega}=0$ and $\varphi(x, T)=$ $\frac{\partial \varphi}{\partial t}(x, T)=0$ in $\Omega$, and integrating by parts over $Q$, we obtain

$$
\begin{aligned}
\int_{Q} v_{n}(x, t) \varphi(x, t) d x d t= & \int_{Q}\left(D_{\mathrm{RL}}^{\alpha} y_{n}(x, t)-\Delta y_{n}(x, t)\right) \varphi(x, t) d x d t \\
= & \int_{\Omega} y^{0} \frac{\partial \varphi}{\partial t}(x, 0) d x \\
& +\int_{Q} y_{n}(x, t)\left(\mathcal{D}_{\mathrm{C}}^{\alpha} \varphi(x, t)-\Delta \varphi(x, t)\right) d x d t
\end{aligned}
$$

because we have (36c) and (36d). Thus, using (40a) and (40c) while passing to the limit in the latter identity, we get

$$
\begin{aligned}
\int_{Q} u^{\gamma}(x, t) \varphi(x, t) d x d t= & \int_{\Omega} y^{0} \frac{\partial \varphi}{\partial t}(x, 0) d x \\
& +\int_{Q} y^{\gamma}(x, t)\left(\mathcal{D}_{\mathrm{C}}^{\alpha} \varphi(x, t)-\Delta \varphi(x, t)\right) d x d t
\end{aligned}
$$

$\forall \varphi \in C^{\infty}(\bar{Q})$ such that $\varphi_{\mid \partial \Omega}=0, \varphi(T)=\frac{\partial \varphi}{\partial t}(T)=0$ in $\Omega$, which, according to Lemma 2.5, can be rewritten as

$$
\begin{aligned}
\int_{Q} u^{\gamma}(x, t) \varphi(x, t) d x d t= & \int_{\Omega} y^{0} \frac{\partial \varphi}{\partial t}(x, 0) d x \\
& +\int_{Q}\left(D_{\mathrm{RL}}^{\alpha} y^{\gamma}(x, t)-\Delta y^{\gamma}(x, t)\right) \varphi(x, t) d x d t \\
& +\left\langle\varphi(x, 0), \frac{\partial}{\partial t} I^{2-\alpha} y^{\gamma}(x, 0)\right\rangle_{H_{0}^{1}(\Omega), H^{-1}(\Omega)} \\
& -\int_{\Omega} \frac{\partial \varphi}{\partial t}(x, 0), I^{2-\alpha} y^{\gamma}(x, 0) d x \\
& -\left\langle y^{\gamma}(\sigma, t), \frac{\partial \varphi}{\partial \nu}(\sigma, t)\right\rangle_{H^{-2}\left((0, T) ; H^{-1 / 2}(\partial \Omega)\right), H_{0}^{2}\left((0, T) ; H^{1 / 2}(\partial \Omega)\right)},
\end{aligned}
$$

$\forall \varphi \in C^{\infty}(\bar{Q})$ such that $\varphi_{\mid \partial \Omega}=0, \varphi(T)=\frac{\partial \varphi}{\partial t}(T)=0$ in $\Omega$.

Using (43), we obtain

$$
\begin{aligned}
0= & \int_{\Omega} y^{0} \frac{\partial \varphi}{\partial t}(x, 0) d x \\
& +\left\langle\varphi(x, 0), \frac{\partial}{\partial t} I^{2-\alpha} y^{\gamma}(x, 0)\right\rangle_{H_{0}^{1}(\Omega), H^{-1}(\Omega)}
\end{aligned}
$$




$$
\begin{aligned}
& -\int_{\Omega} \frac{\partial \varphi}{\partial t}(x, 0) I^{2-\alpha} y^{\gamma}(x, 0) d x \\
& -\left\langle y^{\gamma}(\sigma, t), \frac{\partial \varphi}{\partial \nu}(\sigma, t)\right\rangle_{H^{-2}\left((0, T) ; H^{-1 / 2}(\partial \Omega)\right), H_{0}^{2}\left((0, T) ; H^{1 / 2}(\partial \Omega)\right)},
\end{aligned}
$$

$\forall \varphi \in C^{\infty}(\bar{Q})$ such that $\varphi_{\mid \partial \Omega}=0, \varphi(T)=\frac{\partial \varphi}{\partial t}(T)=0$ in $\Omega$.

Choosing successively in (46) $\varphi$ such that $\varphi(x, 0)=\frac{\partial \varphi}{\partial t}(x, 0)=0$ and $\varphi(x, 0)=0$, we deduce that

$$
\begin{aligned}
& y^{\gamma}(x, t)=0, \quad(x, t) \in \Sigma, \\
& I^{2-\alpha} y^{\gamma}(x, 0)=y^{0}, \quad x \in \Omega,
\end{aligned}
$$

and then

$$
\frac{\partial}{\partial t} I^{2-\alpha} y^{\gamma}(x, 0)=0, \quad x \in \Omega .
$$

In view of (43), (47), (48), and (49), we see that $y^{\gamma}=y^{\gamma}\left(x, t ; u^{\gamma}, 0\right)$ is a solution of (19). Step 2: We show $\zeta_{n}=\zeta\left(x, t ; v_{n}\right)$ converges to $\zeta^{\gamma}=\zeta\left(x, t ; u^{\gamma}\right)$.

In view of $(24), \zeta_{n}=\zeta\left(x, t ; v_{n}\right)$ verifies

$$
\left\{\begin{array}{l}
\mathcal{D}_{\mathrm{C}}^{\alpha} \zeta_{n}-\Delta \zeta_{n}=y\left(v_{n}, 0\right)-y(0,0) \quad \text { in } Q \\
\zeta_{n}=0 \text { on } \Sigma \\
\zeta_{n}(T)=0 \text { in } \Omega \\
\frac{\partial}{\partial t} \zeta_{n}(T)=0 \text { in } \Omega .
\end{array}\right.
$$

Set $z_{n}=y\left(v_{n}, 0\right)-y(0,0)$. In view of (19) and (21), $z_{n}$ verifies

$$
\left\{\begin{array}{l}
D_{\mathrm{RL}}^{\alpha} z_{n}-\Delta z_{n}=v_{n} \quad \text { in } Q \\
z_{n}=0 \quad \text { on } \Sigma \\
I^{2-\alpha} z_{n}(0)=0 \quad \text { in } \Omega \\
\frac{\partial}{\partial t} I^{2-\alpha} z_{n}(0)=0 \quad \text { in } \Omega
\end{array}\right.
$$

It follows, from Theorem 2.6 and (37a), that

$$
\left\|z_{n}\right\|_{L^{2}\left((0, T) ; H_{0}^{1}(\Omega)\right)}=\left\|y_{n}\left(v_{n}, 0\right)-y(0,0)\right\|_{L^{2}\left((0, T) ; H_{0}^{1}(\Omega)\right)} \leq C(\gamma) .
$$

Hence, from Corollary 2.8, we deduce that

$$
\begin{aligned}
\left\|\zeta_{n}\right\|_{C\left([0, T] ; H_{0}^{1}(\Omega)\right)} \leq & C(\gamma), \\
\left\|\frac{\partial}{\partial t} \zeta_{n}\right\|_{C\left([0, T] ; L^{2}(\Omega)\right)} & \leq C(\gamma) .
\end{aligned}
$$

Since the embedding of $C\left([0, T] ; H_{0}^{1}(\Omega)\right)$ into $L^{2}\left((0, T) ; H_{0}^{1}(\Omega)\right)$ and the embedding of $C\left([0, T] ; L^{2}(\Omega)\right)$ into $L^{2}(Q)$ are continuous, we can conclude that there exists $\zeta^{\gamma} \in$ $L^{2}\left((0, T) ; H_{0}^{1}(\Omega)\right)$ such that

$$
\zeta_{n} \rightarrow \zeta^{\gamma} \quad \text { weakly in } L^{2}\left((0, T) ; H_{0}^{1}(\Omega)\right)
$$


Therefore,

$$
\frac{\partial}{\partial t} \zeta_{n} \rightarrow \frac{\partial}{\partial t} \zeta^{\gamma} \quad \text { weakly in } \mathbb{D}^{\prime}(Q)
$$

and, consequently,

$$
\frac{\partial}{\partial t} \zeta_{n} \rightarrow \frac{\partial}{\partial t} \zeta^{\gamma} \quad \text { weakly in } L^{2}(Q)
$$

Since $\zeta^{\gamma} \in L^{2}\left((0, T) ; H_{0}^{1}(\Omega)\right)$ and $\frac{\partial}{\partial t} \zeta^{\gamma} \in L^{2}(Q)$, we see that $\zeta^{\gamma}(0)$ and $\zeta^{\gamma}(T)$ belongs to $L^{2}(\Omega)$. In view of $(50)_{3}$, we have

$$
\zeta^{\gamma}(T)=0 \quad \text { in } \Omega
$$

and in view of (50) ${ }_{4}$ and (52), we set

$$
\frac{\partial}{\partial t} \zeta^{\gamma}(T)=0 \quad \text { in } \Omega
$$

From (37b), we deduce that there exists $\rho \in L^{2}(\Omega)$ such that

$$
\zeta\left(\cdot, 0 ; v_{n}\right) \rightarrow \rho \quad \text { weakly in } L^{2}(\Omega)
$$

Multiplying the first equation of (50) by $\phi \in \mathbb{D}(Q)$ then, using the integration by parts given by Lemma 2.5 , we obtain

$$
\begin{aligned}
& \int_{Q}\left(y\left(x, t ; v_{n}, 0\right)-y(x, t ; 0,0)\right) \phi(x, t) d t d x \\
& =\int_{Q}\left[D_{\mathrm{RL}}^{\alpha} \phi(x, t)-\Delta \phi(x, t)\right] \zeta_{n}(x, t) d t d x
\end{aligned}
$$

Hence, using (40c) and (53) while passing to the limit in the latter identity, we have

$$
\begin{aligned}
& \int_{Q}\left(y\left(x, t ; u^{\gamma}, 0\right)-y(x, t ; 0,0)\right) \phi(x, t) d t d x \\
& \quad=\int_{Q}\left(D_{\mathrm{RL}}^{\alpha} \phi(x, t)-\Delta \phi(x, t)\right) \zeta^{\gamma}(x, t) d t d x, \quad \forall \phi \in \mathbb{D}(Q),
\end{aligned}
$$

which by using again Lemma 2.5 gives

$$
\begin{aligned}
& \int_{Q}\left(y\left(x, t ; u^{\gamma}, 0\right)-y(x, t ; 0,0)\right) \phi(x, t) d t d x \\
& \quad=\int_{Q}\left(\mathcal{D}_{\mathrm{C}}^{\alpha} \zeta^{\gamma}(x, t)-\Delta \zeta^{\gamma}(x, t)\right) \phi(x, t) d t d x, \quad \forall \phi \in \mathbb{D}(Q) .
\end{aligned}
$$

This implies that

$$
\mathcal{D}_{\mathrm{C}}^{\alpha} \zeta^{\gamma}-\Delta \zeta^{\gamma}=y\left(u^{\gamma}, 0\right)-y(0,0) \text { in } Q
$$


Now, if we multiply the first equation of (50) by $\phi \in \mathcal{C}^{\infty}(\bar{Q})$ with $\phi_{\mid \partial \Omega}=0$ and $I^{2-\alpha} \phi(0)=0$ in $\Omega$ and integrating by parts over $Q$, we obtain

$$
\begin{aligned}
& \int_{Q}\left(y_{n}(x, t)-y(x, t ; 0,0)\right) \phi(x, t) d t d x \\
& \quad=\int_{Q}\left(\mathcal{D}_{\mathrm{C}}^{\alpha} \zeta_{n}(x, t)-\Delta \zeta_{n}(x, t)\right) \phi(x, t) d t d x \\
& \quad=\int_{Q}\left(D_{\mathrm{RL}}^{\alpha} \phi(x, t)-\Delta \phi(x, t)\right) \zeta_{n}(x, t) d t d x+\int_{\Omega} \zeta\left(x, 0, v_{n}\right) \frac{\partial}{\partial t} I^{2-\alpha} \phi(0) d x .
\end{aligned}
$$

Using (40c), (53), and (57) while passing the latter identity to the limit, we obtain

$$
\begin{aligned}
& \int_{Q}\left(y^{\gamma}(x, t)-y(x, t ; 0,0)\right) \phi(x, t) d t d x \\
& =\int_{Q}\left(D_{\mathrm{RL}}^{\alpha} \phi(x, t)-\Delta \phi(x, t)\right) \zeta^{\gamma}(x, t) d t d x+\int_{\Omega} \rho \frac{\partial}{\partial t} I^{2-\alpha} \phi(0) d x, \\
& \quad \forall \phi \in \mathcal{C}^{\infty}(\bar{Q}) \text { such that } \phi_{\mid \partial \Omega}=0, I^{2-\alpha} \phi(0)=0 \text { in } \Omega,
\end{aligned}
$$

which by using again Lemma 2.5, (55), (56), and (59) gives

$$
\begin{aligned}
& -\int_{\Sigma} \frac{\partial}{\partial \nu} \phi(\sigma, t) \zeta^{\gamma}(\sigma, t) d \sigma d t+\int_{\Omega} \rho(x) \frac{\partial}{\partial t} I^{2-\alpha} \phi(0) d x \\
& =\int_{\Omega} \zeta^{\gamma}(0) \frac{\partial}{\partial t} I^{2-\alpha} \phi(0) d x \\
& \quad \forall \phi \in \mathcal{C}^{\infty}(\bar{Q}) \text { such that } \phi_{\mid \partial \Omega}=0, I^{2-\alpha} \phi(0)=0 \text { in } \Omega .
\end{aligned}
$$

Hence, choosing $\phi \in \mathcal{C}^{\infty}(\bar{Q})$, such that $\phi_{\mid \partial \Omega}=0, I^{2-\alpha} \phi(0)=\frac{\partial}{\partial t} I^{2-\alpha} \phi(0)=0$, we get

$$
\zeta^{\gamma}=0 \quad \text { on } \Sigma
$$

and then

$$
\zeta^{\gamma}(0)=\rho \quad \text { in } \Omega
$$

In view of (55), (56), (59), and (61), we see that $\zeta^{\gamma}=\zeta\left(u^{\gamma}\right)$ is a solution of

$$
\left\{\begin{array}{l}
\mathcal{D}_{\mathrm{C}}^{\alpha} \zeta^{\gamma}-\Delta \zeta^{\gamma}=y\left(u^{\gamma}, 0\right)-y(0,0) \text { in } Q \\
\zeta^{\gamma}=0 \text { on } \Sigma \\
\zeta^{\gamma}(T)=0 \text { in } \Omega \\
\frac{\partial \zeta^{\gamma}}{\partial t}(T)=0 \text { in } \Omega
\end{array}\right.
$$

Moreover, using (62), equation (57) becomes

$$
\zeta\left(\cdot, 0 ; v_{n}\right) \rightarrow \zeta^{\gamma}(0)=\zeta\left(\cdot, 0 ; u^{\gamma}\right) \quad \text { weakly in } L^{2}(\Omega)
$$

Step 3: The function $v \rightarrow J_{\gamma}(v)$ being lower semi-continuous, we have

$$
J_{\gamma}\left(u^{\gamma}\right) \leq \liminf _{n \rightarrow \infty} J_{\gamma}\left(v_{n}\right)
$$


which in view of (35) implies that

$$
J_{\gamma}\left(u^{\gamma}\right)=\inf _{v \in L^{2}(Q)} J_{\gamma}(v)
$$

The uniqueness of $u^{\gamma}$ comes from the fact that the functional $J_{\gamma}$ is strictly convex.

Theorem 3.4 For any $\gamma>0$, let $u^{\gamma}$ be the low-regret control. Then there exist $q^{\gamma} \in$ $L^{2}\left((0, T) ; H_{0}^{1}(\Omega)\right)$ and $p^{\gamma} \in \mathcal{C}\left([0, T] ; H_{0}^{1}(\Omega)\right)$ such that $\left(u^{\gamma}, y^{\gamma}=y^{\gamma}\left(u^{\gamma}, 0\right), q^{\gamma}, p^{\gamma}\right)$ satisfies the following optimality system:

$$
\begin{aligned}
& \left\{\begin{array}{l}
D_{\mathrm{RL}}^{\alpha} y^{\gamma}-\Delta y^{\gamma}=u^{\gamma} \quad \text { in } Q, \\
y^{\gamma}=0 \text { on } \Sigma, \\
I^{2-\alpha} y^{\gamma}(0)=y^{0} \text { in } \Omega, \\
\frac{\partial}{\partial t} I^{2-\alpha} y^{\gamma}(0)=0 \text { in } \Omega,
\end{array}\right. \\
& \left\{\begin{array}{l}
D_{\mathrm{RL}}^{\alpha} q^{\gamma}-\Delta q^{\gamma}=0 \text { in } Q, \\
q^{\gamma}=0 \text { on } \Sigma, \\
I^{2-\alpha} q^{\gamma}(0)=0 \text { in } \Omega, \\
\frac{\partial}{\partial t} I^{2-\alpha} q^{\gamma}(0)=\frac{1}{\sqrt{\gamma}} \zeta\left(0 ; u^{\gamma}\right) \quad \text { in } \Omega,
\end{array}\right. \\
& \left\{\begin{array}{l}
\mathcal{D}_{\mathrm{C}}^{\alpha} p^{\gamma}-\Delta p^{\gamma}=y^{\gamma}-z_{d}+\frac{1}{\sqrt{\gamma}} q^{\gamma} \quad \text { in } Q, \\
p^{\gamma}=0 \text { on } \Sigma, \\
p^{\gamma}(T)=0 \text { in } \Omega, \\
\frac{\partial p^{\gamma}}{\partial t}(T)=0 \text { in } \Omega,
\end{array}\right.
\end{aligned}
$$

and

$$
N u^{\gamma}+p^{\gamma}=0 \quad \text { in } Q
$$

Proof Equations (43), (47), (48), and (49) give (65). To characterize the low-regret control $u^{\gamma}$, we use the Euler-Lagrange optimality conditions:

$$
\left.\frac{d}{d k} J_{\gamma}\left(u^{\gamma}+k\left(v-u^{\gamma}\right)\right)\right|_{k=0}=0, \quad \forall v \in L^{2}(Q) .
$$

After some calculations, we obtain

$$
\begin{gathered}
\int_{Q}\left(y\left(u^{\gamma}, 0\right)-z_{d}\right)\left(y(v, 0)-y\left(u^{\gamma}, 0\right)\right) d t d x+\int_{Q} N u^{\gamma}\left(v-u^{\gamma}\right) d t d x \\
+\frac{1}{\gamma} \int_{\Omega}\left(\zeta\left(x, 0 ; u^{\gamma}\right), \zeta\left(x, 0 ; v-u^{\gamma}\right)\right) d x=0, \quad \forall v \in L^{2}(Q),
\end{gathered}
$$

where from (24), $\zeta\left(v-u^{\gamma}\right)=\zeta\left(x, t ; v-u^{\gamma}\right) \in C\left([0, T] ; H_{0}^{1}(\Omega)\right)$ is a solution of

$$
\left\{\begin{array}{l}
\mathcal{D}^{\alpha} \zeta\left(v-u^{\gamma}\right)-\Delta \zeta\left(v-u^{\gamma}\right)=y(v, 0)-y^{\gamma}\left(u^{\gamma}, 0\right) \quad \text { in } Q \\
\zeta\left(v-u^{\gamma}\right)=0 \quad \text { on } \Sigma \\
\zeta\left(T ; v-u^{\gamma}\right)=0 \quad \text { in } \Omega \\
\frac{\partial \zeta}{\partial t}\left(T ; v-u^{\gamma}\right)=0 \quad \text { in } \Omega .
\end{array}\right.
$$


Let $z\left(v-u^{\gamma}\right)=y(x, t ; v, 0)-y^{\gamma}\left(x, t ; u^{\gamma}, 0\right)$ be the state associated to $\left(v-u^{\gamma}\right) \in L^{2}(Q)$. Then in view of (19), $z=z\left(v-u^{\gamma}\right) \in L^{2}\left((0, T) ; H_{0}^{1}(\Omega)\right)$ is a solution of

$$
\left\{\begin{array}{l}
D_{\mathrm{RL}}^{\alpha} z-\Delta z=v-u^{\gamma} \quad \text { in } Q \\
z=0 \text { on } \Sigma \\
I^{2-\alpha} z(0)=0 \text { in } \Omega \\
\frac{\partial}{\partial t} I^{2-\alpha} z(0)=0 \text { in } \Omega .
\end{array}\right.
$$

To interpret (70), we introduce $q^{\gamma}=q^{\gamma}\left(u^{\gamma}, 0\right)$ as a solution of equation (66). As $\frac{1}{\sqrt{\gamma}} \zeta\left(\cdot, 0 ; u^{\gamma}\right) \in L^{2}(\Omega)$, according to Theorem $2.6, q^{\gamma}$ is unique and belongs to $L^{2}((0, T)$; $H_{0}^{1}(\Omega)$ ). Moreover,

$$
\left\|q^{\gamma}\right\|_{L^{2}\left((0, T) ; H_{0}^{1}(\Omega)\right)} \leq \frac{C}{\sqrt{\gamma}}\left\|\zeta\left(0 ; u^{\gamma}\right)\right\|_{L^{2}(\Omega)}
$$

where $C>0$ is a positive constant independent of $\gamma$.

Multiplying the first equation of (71) by $\frac{1}{\sqrt{\gamma}} q^{\gamma}$ and using Lemma 2.5, we obtain

$$
\int_{\Omega} \frac{1}{\gamma} \zeta\left(x, 0 ; v-u^{\gamma}\right) \zeta\left(x, 0 ; u^{\gamma}\right) d x=\int_{Q}\left(y(v, 0)-y\left(u^{\gamma}, 0\right)\right) \frac{1}{\sqrt{\gamma}} q^{\gamma} d t d x
$$

which combining with (70) gives

$$
\begin{gathered}
\int_{Q}\left[y(v, 0)-y\left(u^{\gamma}, 0\right)\right]\left[\left(y\left(u^{\gamma}, 0\right)-z_{d}+\frac{1}{\sqrt{\gamma}} q^{\gamma}\right)\right] d t d x \\
+\int_{Q} N u^{\gamma}\left(v-u^{\gamma}\right) d t d x=0, \quad \forall v \in L^{2}(Q) .
\end{gathered}
$$

Now, let $p^{\gamma}$ verify (67). Then, in view of Corollary $2.8, p^{\gamma} \in C\left([0, T] ; H_{0}^{1}(\Omega)\right)$, and $\frac{\partial}{\partial t} p^{\gamma} \in$ $C\left([0, T] ; L^{2}(\Omega)\right)$ since $y^{\gamma}-z_{d}+\frac{1}{\sqrt{\gamma}} q^{\gamma} \in L^{2}(Q)$.

Thus, multiplying the first equation of (72) by $p^{\gamma}$, a solution of (67), then, utilizing the fractional integration by parts provided by Lemma 2.5 , we conclude

$$
\int_{Q} z\left(v-u^{\gamma}\right)\left(\mathcal{D}_{\mathrm{C}}^{\alpha} p^{\gamma}-\Delta p^{\gamma}\right) d x d t=\int_{Q}\left(v-u^{\gamma}\right) p^{\gamma} d x d t
$$

Replacing in the latter identity $z\left(v-u^{\gamma}\right)$ by $y(x, t ; v, 0)-y^{\gamma}\left(x, t ; u^{\gamma}, 0\right)$, which is a solution of (72), we obtain

$$
\begin{aligned}
& \int_{Q}\left(v-u^{\gamma}\right) p^{\gamma} d x d t \\
& \quad=\int_{Q}\left[y(x, t ; v, 0)-y^{\gamma}\left(x, t ; u^{\gamma}, 0\right)\right]\left[\left(y^{\gamma}\left(u^{\gamma}, 0\right)-z_{d}+\frac{1}{\sqrt{\gamma}} q^{\gamma}\right)\right] d t d x
\end{aligned}
$$

which combining with (74) gives

$$
\int_{Q}\left(N u^{\gamma}+p^{\gamma}\right)\left(v-u^{\gamma}\right) d x d t=0, \quad \forall v \in L^{2}(Q) .
$$

Consequently $N u^{\gamma}+p^{\gamma}=0$ in $Q$. 
Proposition 3.5 For any $\gamma>0$, let $u^{\gamma}$ be the low-regret control. Then $u^{\gamma}$ converges to $u, a$ solution of the no-regret problem (30).

Proof As $u^{\gamma}$ is a solution of (33), we have

$$
J_{\gamma}\left(u^{\gamma}\right) \leq J_{\gamma}(0)=0
$$

because in view of $(24), \zeta(0)=\zeta(x, t ; 0)=0$ in $Q$. It then follows from the definition of $J_{\gamma}$ given by (34) that

$$
\begin{aligned}
& \left\|y\left(u^{\gamma}, 0\right)-z_{d}\right\|_{L^{2}(Q)}^{2}+N\left\|u^{\gamma}\right\|_{L^{2}(Q)}^{2}+\frac{1}{\gamma}\left\|\zeta\left(\cdot, 0 ; u^{\gamma}\right)\right\|_{L^{2}(\Omega)}^{2} \\
& \leq J(0,0)=\left\|y(0,0)-z_{d}\right\|_{L^{2}(Q)}^{2} .
\end{aligned}
$$

Therefore, we deduce that

$$
\begin{aligned}
& \left\|y\left(u^{\gamma}, 0\right)\right\|_{L^{2}(Q)} \leq\left\|y(0,0)-z_{d}\right\|_{L^{2}(Q)}, \\
& \left\|u^{\gamma}\right\|_{L^{2}(Q)} \leq \frac{1}{N}\left\|y(0,0)-z_{d}\right\|_{L^{2}(Q)}, \\
& \left\|\zeta\left(\cdot, 0 ; u^{\gamma}\right)\right\|_{L^{2}(\Omega)} \leq \sqrt{\gamma}\left\|y(0,0)-z_{d}\right\|_{L^{2}(Q)} .
\end{aligned}
$$

Hence from $(75 b)$ and $(65)_{1}$, we have

$$
\left\|D_{\mathrm{RL}}^{\alpha} y\left(u^{\gamma}, 0\right)-\Delta y\left(u^{\gamma}, 0\right)\right\|_{L^{2}(Q)} \leq \frac{1}{N}\left\|y(0,0)-z_{d}\right\|_{L^{2}(Q)} .
$$

Since $y\left(u^{\gamma}, 0\right)$ is solution of $(65)$, we see from Theorem 2.6 that there exists a constant $C$ independent of $\gamma$ such that

$$
\left\|y\left(u^{\gamma}, 0\right)\right\|_{L^{2}\left((0, T) ; H_{0}^{1}(\Omega)\right)} \leq \frac{C}{N}\left\|y(0,0)-z_{d}\right\|_{L^{2}(Q)} .
$$

Thus there exist $u \in L^{2}(Q), y \in L^{2}\left((0, T) ; H_{0}^{1}(\Omega)\right), \delta \in L^{2}(Q)$, and subsequences extracted of $\left(u^{\gamma}\right)$ and $\left(y^{\gamma}\right)$ (still called $\left(u^{\gamma}\right)$ and $\left.\left(y^{\gamma}\right)\right)$ such that

$$
\begin{aligned}
& u^{\gamma} \rightarrow u \quad \text { weakly in } L^{2}(Q), \\
& y^{\gamma} \rightarrow y \quad \text { weakly in } L^{2}\left((0, T) ; H_{0}^{1}(\Omega)\right), \\
& D_{\mathrm{RL}}^{\alpha} y^{\gamma}-\Delta y^{\gamma} \rightarrow \delta \quad \text { weakly in } L^{2}(Q) .
\end{aligned}
$$

If we proceed as in pp.10 to 14 , using (78a)-(78c), we show that $y=y(x, t ; u, 0)$ is such that

$$
\left\{\begin{array}{l}
D_{\mathrm{RL}}^{\alpha} y-\Delta y=u \quad \text { in } Q \\
y=0 \text { on } \Sigma, \\
I^{2-\alpha} y(x, 0)=y^{0} \quad \text { in } \Omega, \\
\frac{\partial}{\partial t} I^{2-\alpha} y(x, 0)=0 \quad \text { in } \Omega
\end{array}\right.
$$


and $\zeta=\zeta(x, t ; u) \in \mathcal{C}\left([0, T] ; H_{0}^{1}(\Omega)\right)$ is a solution of

$$
\left\{\begin{array}{l}
\mathcal{D}^{\alpha} \zeta-\Delta \zeta=y(u, 0)-y(0,0) \quad \text { in } Q \\
\zeta=0 \text { on } \Sigma \\
\zeta(T)=0 \text { in } \Omega \\
\frac{\partial \zeta}{\partial t}(T)=0 \text { in } \Omega
\end{array}\right.
$$

Moreover, in view of $(75 \mathrm{c})$, we have

$$
\zeta\left(\cdot, 0 ; u^{\gamma}\right) \rightarrow \zeta(\cdot, 0 ; u)=0 \quad \text { strongly in } L^{2}(\Omega)
$$

Consequently, $\int_{\Omega} g \zeta(x, 0 ; u) d x=0$.

This implies that $u$ is solution of the no-regret control problem (30).

Theorem 3.6 Let us consider $u=\lim _{\gamma \rightarrow 0} u^{\gamma}$ be the no-regret control corresponding to the state $y(u, 0)$. Then there exist $q \in L^{2}\left((0, T) ; H_{0}^{1}(\Omega)\right)$ and $p \in \mathcal{C}\left([0, T] ; H_{0}^{1}(\Omega)\right)$ in such a way that $(u, y=y(u, 0), q, p)$ fulfills the following optimality system:

$$
\begin{aligned}
& \left\{\begin{array}{l}
D_{\mathrm{RL}}^{\alpha} y-\Delta y=u \text { in } Q, \\
y=0 \text { on } \Sigma, \\
I^{2-\alpha} y(0)=y^{0} \text { in } \Omega, \\
\frac{\partial}{\partial t} I^{2-\alpha} y(0)=0 \text { in } \Omega,
\end{array}\right. \\
& \left\{\begin{array}{l}
D_{\mathrm{RL}}^{\alpha} q-\Delta q=0 \text { in } Q, \\
q=0 \text { on } \Sigma, \\
I^{2-\alpha} q(0)=0 \text { in } \Omega, \\
\frac{\partial}{\partial t} I^{2-\alpha} q(0)=\tau_{1} \quad \text { in } \Omega,
\end{array}\right. \\
& \left\{\begin{array}{l}
\mathcal{D}_{\mathrm{C}}^{\alpha} p-\Delta p=y(u, 0)-z_{d}+\tau_{2} \quad \text { in } Q, \\
p=0 \text { on } \Sigma, \\
p(T)=0 \text { in } \Omega, \\
\frac{\partial p}{\partial t}(T)=0 \text { in } \Omega,
\end{array}\right.
\end{aligned}
$$

and

$$
N u+p=0 \quad \text { in } Q .
$$

Proof We have (82) (see system (79)).

From $(75 \mathrm{c})$, we get

$$
\left\|\frac{1}{\sqrt{\gamma}} \zeta\left(0 ; u^{\gamma}\right)\right\|_{L^{2}(\Omega)} \leq\left\|y(0,0)-z_{d}\right\|_{L^{2}(Q)} .
$$

Consequently, equation (73) becomes

$$
\left\|q^{\gamma}\right\|_{L^{2}\left((0, T) ; H_{0}^{1}(\Omega)\right)} \leq C\left\|y(0,0)-z_{d}\right\|_{L^{2}(Q)} .
$$

Thus, there exist $\tau_{1} \in L^{2}(\Omega)$ and $q \in L^{2}\left(0, T ; H_{0}^{1}(\Omega)\right)$ such that

$$
\frac{1}{\sqrt{\gamma}} \zeta\left(\cdot, 0 ; u^{\gamma}\right) \rightarrow \tau_{1} \quad \text { weakly in } L^{2}(\Omega),
$$




$$
q^{\gamma} \rightarrow q \quad \text { weakly in } L^{2}\left((0, T) ; H_{0}^{1}(\Omega)\right) .
$$

Using (87) and (88) while passing to the limit in (66), we show as for the convergence of $y_{n}=y\left(v_{n}, 0\right)$ (see pp.10 to 12 ) that $q$ satisfies (83).

From (68) and (75b), we have

$$
\left\|p^{\gamma}\right\|_{L^{2}(Q)} \leq\left\|y(0,0)-z_{d}\right\|_{L^{2}(Q)} .
$$

Therefore there exists $p \in L^{2}(Q)$ such that

$$
p^{\gamma} \rightarrow p \quad \text { weakly in } L^{2}(Q) \text {. }
$$

In view of (67) and (75a), we know that there exist $\tau_{2} \in L^{2}(Q)$ such that

$$
\frac{1}{\sqrt{\gamma}} q^{\gamma} \rightarrow \tau_{2} \quad \text { weakly in } L^{2}(Q) \text {. }
$$

Then we prove as for the convergence of $\zeta_{n}=\zeta\left(x, t ; v_{n}\right)$ (see pp.12 to 14) that $p$ is solution of (84). Using (75b) and (89) while passing to the limit in (68), we conclude (85).

\section{Conclusions}

We study an optimal control problem associated to a fractional wave equation involving Riemann-Liouville fractional derivative and with incomplete data. Actually, the initial condition is missing. In order to solve the problem, we assume that the missing data belongs to an infinite dimensional space. Using the notions of no-regret and low-regret controls, we show that when $3 / 2 \leq \alpha \leq 2$, such a control exists and is unique. Then we give the singular optimality system that characterizes the control.

\section{Competing interests}

The authors declare that they have no competing interests.

\section{Authors' contributions}

All authors contributed equally to the writing of this paper. All authors read and approved the final manuscript.

\section{Author details}

${ }^{1}$ Department of Mathematics, Faculty of Arts and Sciences, Çankaya University, Ankara, 06530, Turkey. ${ }^{2}$ Institute of Space Sciences, Magurele-Bucharest, Romania. ${ }^{3}$ Université des Antilles et de la Guyane, Campus Fouillole, 97159, Pointe-à-Pitre, Guadeloupe (FWI), France. ${ }^{4}$ Laboratoire MAINEGE, Université Ouaga 3S, 06 BP 10347, Ouagadougou 06, Burkina Faso.

\section{Acknowledgements}

The authors are grateful to the referees for their valuable suggestions. The work was supported by the Région Martinique (FWI).

Received: 18 June 2016 Accepted: 12 September 2016 Published online: 21 September 2016

\section{References}

1. Mophou, G, Joseph, C: Optimal control with final observation of a fractional diffusion wave equation. Dyn. Contin. Discrete Impuls. Syst. (accepted)

2. Lions, JL: Contrôle optimal de systèmes gouvernés par des équations aux dérivées partielles. Dunod, Paris (1968)

3. Biswas, RK, Sen, S: Free final time fractional optimal control problems. J. Franklin Inst. 351, 941-951 (2014)

4. Guo, TL: The necessary conditions of fractional optimal control in the sense of Caputo. J. Optim. Theory Appl. 156, 115-126 (2013)

5. Mophou, GM: Optimal control of fractional diffusion equation. Comput. Math. Appl. 61, 68-78 (2011)

6. Agrawal, OP: A general formulation and solution scheme for fractional optimal control problems. Nonlinear Dyn. 38, 323-337 (2004)

7. Agrawal, OP: Formulation of Euler-Lagrange equations for fractional variational problems. J. Math. Anal. 272, 368-379 (2002) 
8. Frederico, GFF, Torres, DFM: Fractional optimal control in the sense of Caputo and the fractional Noether's theorem. Int. Math. Forum 3(10), 479-493 (2008)

9. Ozdemir, N, Karadeniz, D, Skender, BB: Fractional optimal control problem of a distributed system in cylindrical coordinates. Phys. Lett. Aa 373, 221-226 (2009)

10. Baleanu, D, Machado, JAT, Luo, ACJ: Fractional Dynamics and Control. Springer, New York (2012)

11. Malinowska, AB, Odzijewicz, T, Torres, DFM: Advanced Methods in the Fractional Calculus of Variations. SpringerBriefs in Applied Sciences and Technology. Springer, Berlin (2015)

12. Lions, JL: Contrôle à moindres regrets des systèmes distribués. C. R. Acad. Sci. Paris, Ser. I Math. 315, 1253-1257 (1992)

13. Lions, JL: No-Regret and Low-Regret Control: Environment, Economics and Their Mathematical Models. Masson, Paris (1994)

14. Nakoulima, O, Omrane, A, Velin, J: Perturbations à moindres regrets dans les systèmes distribués à données manquantes. C. R. Acad. Sci. Paris, Sér. I Math. 330, 801-806 (2000)

15. Nakoulima, O, Omrane, A, Velin, J: No-regret control for nonlinear distributed systems with incomplete data. J. Math. Pures Appl. 81, 1161-1189 (2002)

16. Jacob, B, Omrane, A: Optimal control for age-structured population dynamics of incomplete data. J. Math. Anal. Appl. 370(1), $42-48(2010)$

17. Mophou, G: Optimal control for fractional diffusion equations with incomplete data. J. Optim. Theory Appl. (2015). doi:10.1007/s10957-015-0817-6

18. Nakoulima, O, Omrane, A, Dorville, R: Low-regret control of singular distributed systems: the ill-posed backwards heat problem. Appl. Math. Lett. 17, 549-552 (2004)

19. Gabay, D, Lions, JL: Décisions stratégiques à moindres regrets. C. R. Acad. Sci. Paris, Sér. I 319, 1249-1256 (1994)

20. Lions, JL: Least regret control, virtual control and decomposition methods. Math. Model. Numer. Anal. 34(2), 409-418 (2000)

21. Nakoulima, O, Omrane, A, Velin, J: Low-regret perturbations in distributed systems with incomplete data. SIAM J. Control Optim. 42(4), 1167-1184 (2003)

22. Nakoulima, O, Omrane, A, Dorville, R: Contrôle optimal pour les problèmes de contrôlabilité des systèmes distribués à données manquantes. C. R. Acad. Sci. Paris, Sér. I 338, 921-924 (2004)

23. Lions, JL: Duality Arguments for Multi Agents Least-Regret Control. Collège de France, Paris (1999)

24. Oldham, KB, Spanier, J: The Fractional Calculus. Academic Press, New York (1974)

25. Samko, SG, Kilbas, AA, Marichev, Ol: Fractional Integral and Derivatives: Theory and Applications. Gordon \& Breach, Yverdon (1993)

26. Kilbas, AA, Srivastava, HM, Trujillo, Jj: Theory and Applications of Fractional Differential Equations. Elsevier, Amsterdam (2006)

27. Podlubny, I: Fractional Differential Equations. Academic Press, San Diego (1999)

28. Sakamoto, K, Yamamoto, M: Initial value/boundary value problems for fractional diffusion-wave equations and applications to some inverse problems. J. Math. Anal. Appl. 382, 426-447 (2011)

\section{Submit your manuscript to a SpringerOpen ${ }^{\ominus}$ journal and benefit from:}

- Convenient online submission

Rigorous peer review

- Immediate publication on acceptance

- Open access: articles freely available online

High visibility within the field

- Retaining the copyright to your article 